\title{
Five-year mortality rate in an ophthalmic ward in Zimbabwe
}

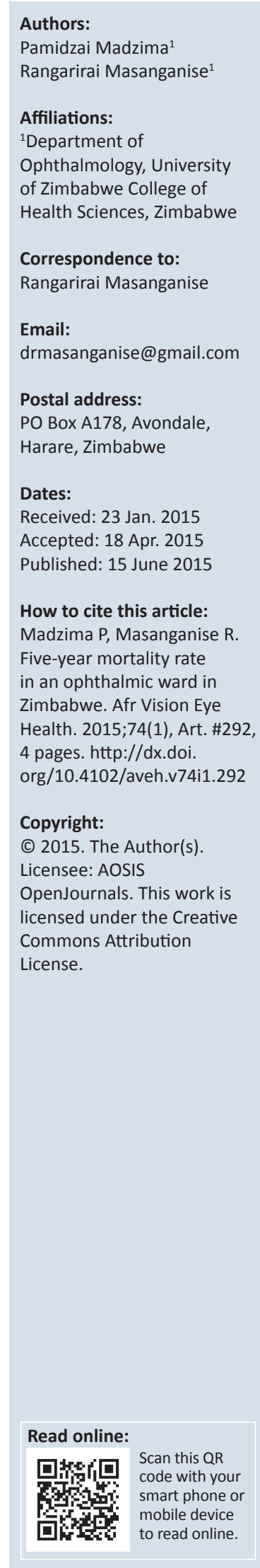

Objective: To determine the mortality rate in the ophthalmic ward at the Sekuru Kaguvi Hospital Eye Unit (SKHEU), Zimbabwe, from January 2009 to December 2013 and to identify the causes of death.

Design: A hospital-based clinical audit.

Methods: Data from completed hospital death notification forms and medical records of patients who had died in the ward during the review period were collected that included age, gender, diagnoses, date of admission, date of death and cause of death. The data were analysed using Epi Info7 software.

Setting: The audit was conducted at SKHEU, the largest tertiary eye unit in Zimbabwe. It comprises an ophthalmic ward with 30 beds and caters for about 900 patients per year. Patients are admitted via the Out-patients Department where about 30000 patients are seen yearly. SKHEU is part of the Parirenyatwa Group of Hospitals, which is one of the four major referral hospitals in Zimbabwe.

Results: Of the 4722 ophthalmic admissions at SKHEU during the 5-year study period, there were $15(0.3 \%)$ deaths, with a male:female ratio of $3: 2$ giving a mortality rate of $0.3 \%$ and an average of 3 deaths per year. The highest number of deaths was in 2009 when 7 deaths occurred, whilst the lowest number was 1 death in 2010 and 1 in 2013. Of the 15 deaths, $4(26.7 \%)$ were children $<12$ years old and $11(73.3 \%)$ were adults; of whom $12(80 \%)$ patients had orbital malignancies, $2(13.3 \%)$ had orbital cellulitis and $1(6.7 \%)$ had ocular trauma. The orbital malignancies included ocular surface squamous neoplasia (OSSN), retinoblastoma and non-Hodgkins lymphoma (NHL). The most common probable cause of death was OSSN which accounted for $9(60 \%)$ of the deaths.

Conclusion: The mortality rate at SKHEU was $0.3 \%$, with approximately 3 deaths occurring per year. The most common attributable cause of death was OSSN.

\section{Introduction}

Mortalities arising from pathologies of the eye are relatively uncommon compared with other medical specialties. ${ }^{1,2}$ In a resource-constrained low- and medium-income setting, ophthalmic mortality rates are higher and often attributable to avoidable and potentially treatable causes such as severe infections and orbito-ocular malignancies. ${ }^{1,2,3,4,5,6}$ In contrast, in developed economies where facilities are available and access barriers to optimal eye care have been largely overcome, the rates are lower and mainly owing to unavoidable causes. ${ }^{2,7,8}$ The mortality rate of a health institution may be an indicator of its performance or a representation of the burden of fatal conditions that the institution is faced with. ${ }^{9}$ It is of great importance to frequently audit the mortality rate of a health centre so that appropriate interventions to lower the rate are implemented in the health delivery system.

The objectives of the present study were to determine the mortality rate in the ophthalmic ward at the Sekuru Kaguvi Hospital Eye Unit (SKHEU), identify the possible causes of death and suggest interventions for reducing mortality in the eye ward.

\section{Methodology}

Permission to conduct the study and ethical clearance were obtained from the hospital and the local Institution Review Board and the Joint Research and Ethics Committee. The completed hospital death notification forms (form BD12) of patients who had died in the ward between 01 January 2009 and 31 December 2013 were reviewed. The data collected included the deceased's name, age, gender, diagnosis, date of admission and date of death. The information gathered was used to retrieve admission records which were reviewed. 


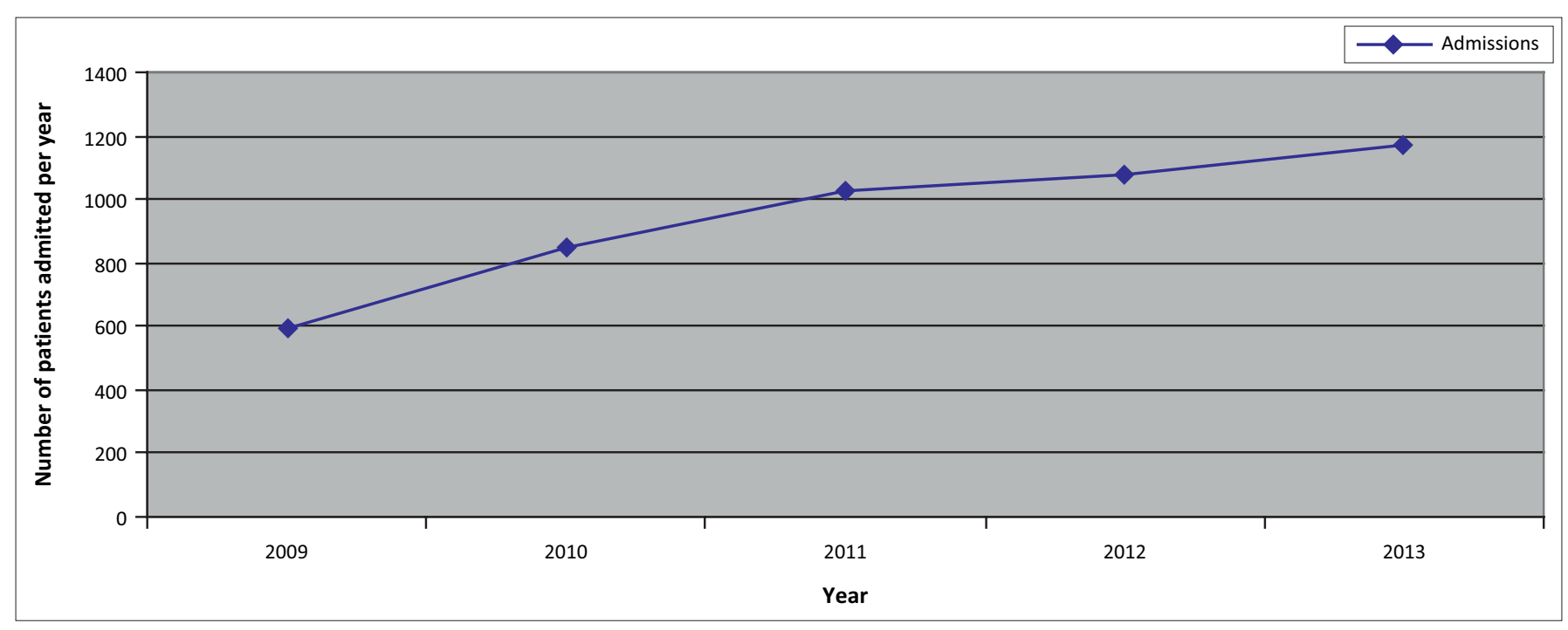

Source: SKHEU hospital admissions register

FIGURE 1: Annual admissions during the study period.

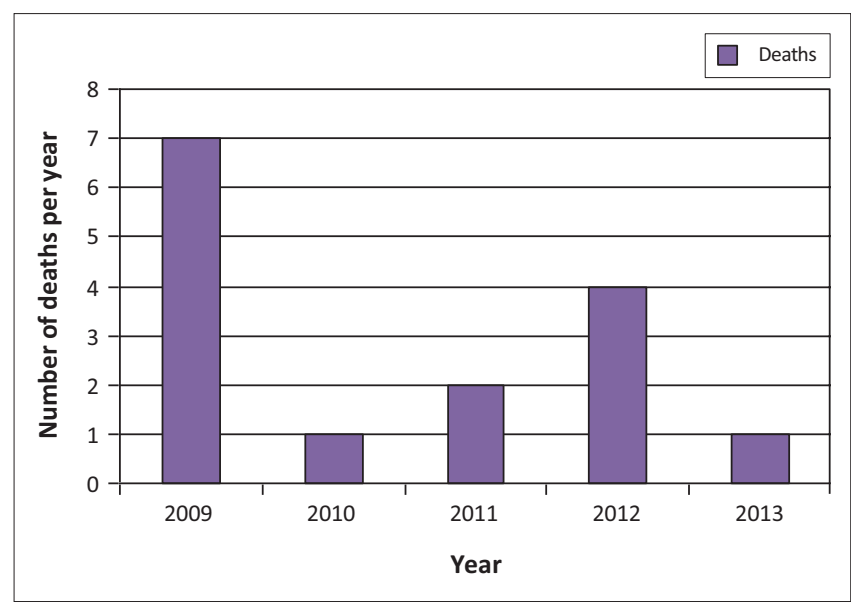

Source: SKHEU hospital death register.

FIGURE 2: Distribution of deaths per year.

The admission register was used to collate annual admissions during the study period. The data were analysed using Epi Info7 software.

\section{Results}

Of the 4722 ophthalmic admissions at SKHEU during the 5 -year study period, there were $15(0.3 \%)$ deaths. The 5-year mortality rate at SKHEU was therefore $0.3 \%$, with an average of 3 deaths per year. Figure 1 shows annual admissions during the study period, and Figure 2 shows the number of deaths per year in the ward during the same period.

There were 9 male patients and 6 female patients, giving a male:female gender ratio of 3:2.

The ages of the deceased ranged from 1 month to 94 years, with a median age of 38 years. Of the 15 deaths, $4(26.7 \%)$ were children $<12$ years old and $11(73.3 \%)$ were adults. Of the 4 children, 2 (50\%) had retinoblastoma and $2(50 \%)$ had orbital cellulitis.

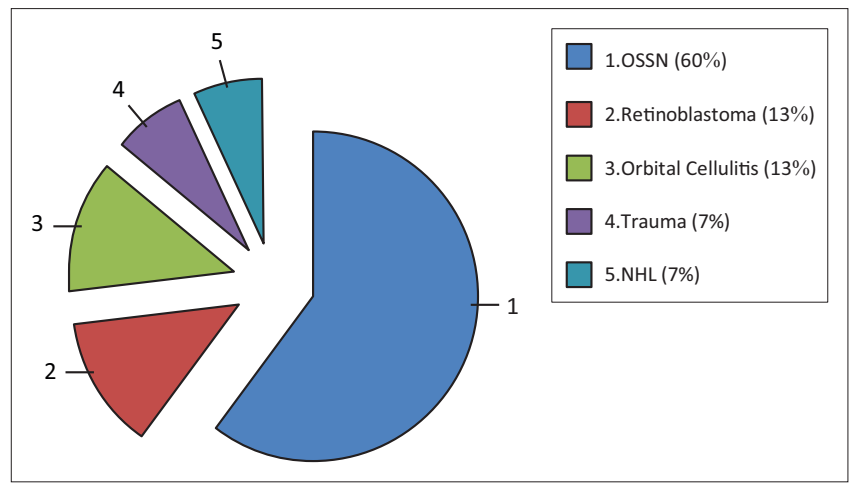

NHL, non-Hodgkin's lymphoma; OSSN, ocular surface squamous neoplasia. FIGURE 3: Attributed causes of death.

Overall, $12 / 15(80 \%)$ patients had orbital malignancies, $2 / 15(13.3 \%)$ had orbital cellulitis and 1/15 (6.7\%) had ocular trauma with a ruptured globe. All the orbital malignancies were primary tumours arising from local orbital structures. Ocular surface squamous neoplasia (OSSN) was the most common probable cause of death, accounting for $9 / 15(60 \%)$ deaths. The other orbital malignancies were retinoblastoma (2/15 [13.3\%]) and nonHodgkins lymphoma (1/15 [6.7\%]). The probable causes of death are listed in Figure 3.

\section{Discussion}

The mortality rate of $0.3 \%$ observed in the ophthalmic ward at SKHEU over the period 2009-2013 is similar to that reported at the same institution from 1984 to 2008 by Masanganise et al. ${ }^{10}$ and has therefore remained constant over the past 30 years. This statistic could suggest that service delivery at SKHEU has been consistent for the past 30 years. The mortality rate at SKHEU is comparable to the rates of $0.3 \%$ and $0.4 \%$ reported respectively by Eze et al. ${ }^{2}$ and Fafowora et al. ${ }^{1}$ from two independent tertiary eye centres in Nigeria. In contrast, Ayrton et al. ${ }^{11}$ reported a mortality rate of zero in the ophthalmic ward of a tertiary eye centre in 
southern Sudan. The overall low ophthalmic mortality rate documented in the present study and previously reported at SKHEU and elsewhere is consistent with the established lower mortality rate among ophthalmic inpatients compared with other medical specialties. ${ }^{12,13,14,15}$

The highest number of deaths at SKHEU occurred in 2009, at a time that the country was experiencing an epidemic of OSSN and the patients presented with advanced tumours that were challenging to manage. ${ }^{10}$ During that period, the country was also undergoing economic challenges that adversely affected health service delivery, and the HIV/AIDs epidemic was at a peak. Subsequently, there has been a general decline in the number of deaths at SKHEU even though admissions have been increasing each year. This finding could be suggestive of an improvement in the hospital's service delivery, with more patients being managed early and appropriately as evidenced by shorter waiting periods for surgery, better medical stock levels in the hospital pharmacy, and less advanced disease being seen at presentation.

Orbital malignancies were the most common cause of mortality at SKHEU. Eze et al. ${ }^{2}$ reported similar findings in Nigeria. OSSN was the leading cause of death at SKHEU during the period 1984-2008 and remains the leading cause to date. ${ }^{10}$ This could be an indication that the hospital is still receiving a large number of advanced OSSN cases where curative treatment is not yet well established, and may also be a reflection of the poor inter-specialty referral system at the hospital because patients with advanced and inoperable tumours remain in the ophthalmic ward instead of being transferred to an oncology ward.

Infection-related ophthalmic mortality usually results from overwhelming bacterial or fungal orbito-ocular infections associated with orbital, intra-cranial or systemic complications. ${ }^{2,4}$ Orbital cellulitis, as an emergency, becomes fatal if it complicates into septicaemia, cavernous sinus thrombosis, meningitis or brain abscess. ${ }^{16,17}$ The two orbital cellulitis patients were treated promptly; however, they nevertheless died soon after admission, suggesting that they had presented with advanced disease that had already complicated.

Twenty-six per cent $(4 / 15)$ of the deceased were children, and retinoblastoma accounted for $50 \%(2 / 4)$ of these deaths. Zimbabwe has the third-highest incidence of retinoblastoma in the world. In low- and medium-income countries, the mortality rate from retinoblastoma children is higher than that in developed countries. ${ }^{18,19}$ About 300 children are admitted at SKHEU per year, of whom about 20 have retinoblastoma. According to the present study, 2 children died from retinoblastoma over the 5-year period. Therefore, it can be estimated that the mortality rate of retinoblastoma amongst children in the ward at SKHEU is $0.1 \%$.

\section{Conclusion}

The mortality rate in the ophthalmic ward at SKHEU was $0.3 \%$, with an average of 3 deaths occurring per year, and the leading attributable cause of death was OSSN. The present study found that the mortality rate has been steady for the past 30 years.

\section{Recommendations}

The following actions need to be implemented:

- Awareness campaigns and screening programmes to educate the population on presenting symptoms and signs of OSSN and retinoblastoma to promote early detection and early treatment. This would tend to reduce the mortality rate from these malignancies.

- Patients admitted to an ophthalmic ward who develop other comorbidities should be promptly referred and jointly managed by the relevant specialties to prevent ward deaths from non-ophthalmic conditions.

\section{Study limitations}

Autopsies were not performed on the deceased because relatives did not consent for various reasons, some of the reasons being: traditional beliefs about the cause of death, cost implications, delays in processing burial for the deceased, and unclear purposes of autopsy. Lack of supporting evidence from autopsy also presents challenges to confirming the cause of death; hence our use of the term 'probable' for cause of death.

\section{Acknowledgements}

The authors thank the African Vision Research Institute for financial assistance with publishing page fees.

\section{Competing interests}

The authors declare that they have no financial or personal relationships which may have inappropriately influenced them in writing this article.

\section{Authors' contributions}

P.M. (University of Zimbabwe College of Health Sciences) was the researcher, R.M. (University of Zimbabwe College of Health Sciences) was the supervisor of the research project.

\section{References}

1. Fafowora OF, Ubah JN. Mortality on an ophthalmic ward. West Afr J Med. 1999;18:211-213.

2. Eze B, Uche J, Shiweobi J. Ophthalmic mortality in a tertiary eye care centre in south-eastern Nigeria. Int J Ophthalmol Vis Sci. 2009;8:2.

3. Omoti $A E$, Ogbedo E. Ophthalmic mortality in a tertiary centre in Nigeria. Niger Postgrad Med J. 2007;14:54-56.

4. Chaudry IA, Shamsi FA, Elzaridi E, et al. Outcome of treated orbital cellulitis in a tertiary eye care centre in the Middle East. Ophthalmology. 2007;114:345-354. http://dx.doi.org/10.1016/j.ophtha.2006.07.059

5. Chuka-Okosa CM, Uche NJ, Kizor-Akaraiwe NN. Orbito-ocular neoplasms in Enugu, south-eastern Nigeria. West Afr J Med. 2008;27:144-147.

6. Thakur SK, Sah SP, Lakhey M, et al. Primary malignant tumours of eye and adnexa in eastern Nepal. Clin Experiment Ophthalmol. 2003;31:415-417. http://dx.doi. org/10.1046/j.1442-9071.2003.00688.x

7. Rahman I, Mano A, Cook AE, Leatherbarrow B. Mortality following exenteration for malignant tumours of the orbit. Br J Ophthalmol. 2005;89:1445-1448. http:// dx.doi.org/10.1136/bjo.2005.072892 
8. Mckibbin N. The preoperative assessment and investigation of ophthalmic patients. Eye. 1996;10:138-140. http://dx.doi.org/10.1038/eye.1996.25

9. Wu WC, Schachat AP. Transfer from ophthalmology to another service is a marker of high risk medical events. Ophthalmic Surg. 1991;22:7-11.

10. Masanganise R, Mashoko A. Mortality in an ophthalmic ward at a tertiary eye unit in Zimbabwe. Cent Afr J Med. 2008;54:49-51.

11. Ayrton J, Attwood D, Kuron Lado D. A retrospective analysis of mortality distribution at Juba Teaching Hospital in Southern Sudan. SSMJ. 2008;2 1-5.

12. Adekunle $\mathrm{O}$, Olatunde IO, Abdul Lateef RM. Causes and pattern of death in a tertiary health institution in south-western Nigeria. Niger Postgrad Med J. 2008:15:247-250.

13. Sani MU, Mohammed AZ, Bapp A, Borodo MM. A three-year review of mortality patterns in the medical wards of Aminu Kano Teaching Hospital, Kano, Nigeria. Niger Postgrad Med J. 2007;14:347-351.
14. Sanclemente C, Barcons M, Moleiro MA, et al. Hospital mortality in an internal medicine service. An Med Intern. 2004;21:317-321.

15. Ramos Rincon JM, Garcia Ruiperez D, Aliaga Matas F, et al. Specific mortality rates by DRG and main diagnosis according to CIE-9-MC at a level II hospital. Ann Med Intern. 2001;18:510-516.

16. Asensi V, Carton JA, Maradona JA, Arribas JM. Severe orbital cellulitis: therapeutic results in 9 patients and review of literature. Enferm Infecc Microbiol Clin 1996;14:250-254.

17. Herman BW, Forsen JW Jr. Simultaneous intra-cranial and orbital complications of acute rhinosinusitis in children. Int J Paediatr Otolaryngol. 2004;68:619-625. http://dx.doi.org/10.1016/j.ijporl.2003.12.010

18. Parkin DM, Kramarova E, Draper GJ, et al., editors. International incidence of childhood cancer. Volume 2. Lyon: IARC; 1998.

19. Ramasubramanian A, Shields CL. Retinoblastoma. New Delhi: Jaypee Brothers, 2012; p. 11-13. http://dx.doi.org/10.5005/jp/books/11629 